\title{
CLOSED-ANGLE GLAUCOMA* SIGNIFICANCE OF SECTORAL VARIATIONS IN ANGLE DEPTH BY
}

\author{
CALBERT I. PHILLIPS \\ From the Glaucoma Clinic, Institute of Ophthalmology, University of London \\ Director of Research: Sir Stewart Duke-Elder
}

THE authors of the published work on the subject of closed-angle (congestive) glaucoma are agreed that, although unstable vascular or nervous systems may exist in the patients and play a part of undetermined importance in the disease, the vast majority of affected eyes show an inherited shallow anterior chamber (Törnquist, 1953), and a forward ballooning of the iris produced by resistance to the flow of aqueous between lens and iris. This resistance is due to the close contact between these two structures imposed on them by the forward position of the anterior surface of the lens. As the years pass and the lens increases in size, so the ballooned iris is pushed forwards and its periphery gradually approaches the trabeculae; an " attack of glaucoma " may occur as a result of pupillary dilatation, a change in position of the iris root, a change in the curvature or position of the lens, or any factor which alters the pressure on the two surfaces of the iris so that it is pushed, or pulled, on to the trabeculae over a wide enough area to embarrass drainage of the aqueous humour. It is also generally agreed that a peripheral iridectomy, or even an iridotomy, will relieve the symptoms by allowing the aqueous to by-pass the pupil so that the angle will open as the iris bombe disappears: the classical broad iridectomy achieves the same result but adds an unnecessary trauma to the sphincter pupillae.

Evidence has been adduced to show that goniosynechiae in closed-angle glaucoma occur especially in the superior half of the angle of the anterior chamber (Phillips, 1956), a finding which confirms the clinical impression that the angle is almost invariably shallower above than below. Barkan $(1936,1938)$ has observed that both the normal and the narrow angle show this phenomenon and suggests that it may be due to the weight of the column of aqueous in the anterior chamber. François (1955) also remarked this anatomical peculiarity. The purpose of this communication is to draw attention to the phenomenon and to discuss the part it plays in the natural history of closed-angle glaucoma.

\section{Irido-Corneal Contact}

Fig. 1 (opposite) is a drawing of the angle of an eye which shows the variation in depth of its angle.

* Received for publication December 5, 1955. 
Case 1, a woman aged 37, had noticed in her right eye intermittent, very transient haloes accompanied by discomfort and blurring of vision for 3 years. This right eye gave a markedly positive dark-room test, and it is reasonable to conclude that it was in the pre-glaucomatous stage of closed-angle glaucoma.

Even if allowance be made for the fact that pigmentation occurs more markedly below, thus making the trabeculae more easily visible in this region, it is obvious that no trabeculae can be seen above, where the iris, at least in the twelve o'clock position, is in contact with the cornea. This is the usual finding in eyes in the prodromal stage of closed-angle glaucoma. If the contact covers the whole antero-posterior extent of the trabeculae in this région, 'drainage of aqueous will be greatly impeded, if not completely prevented. If the angle is traced downwards nasally and temporally, it is difficult to define an exact point where contact between iris and correa is lost. In Fig. 1 this does not seem to have occurred before the 3 and 9 o'clock positions have been reached, so that only the lowermost part of the angle is available for drainage. The history suggests that this eye has reached the dangerous stage where a slight increase in the area of the trabeculae covered by the iris will result in a rise of tension.

Fig. 1.-Gonioscopic appearances of the right eye of Case 1 , a woman aged 37 , who had the premonitory symptoms of closed-angle glaucoma. The angle is almost certainly closed above but is open below; nasally and temporally it is extremely narrow, if not closed.

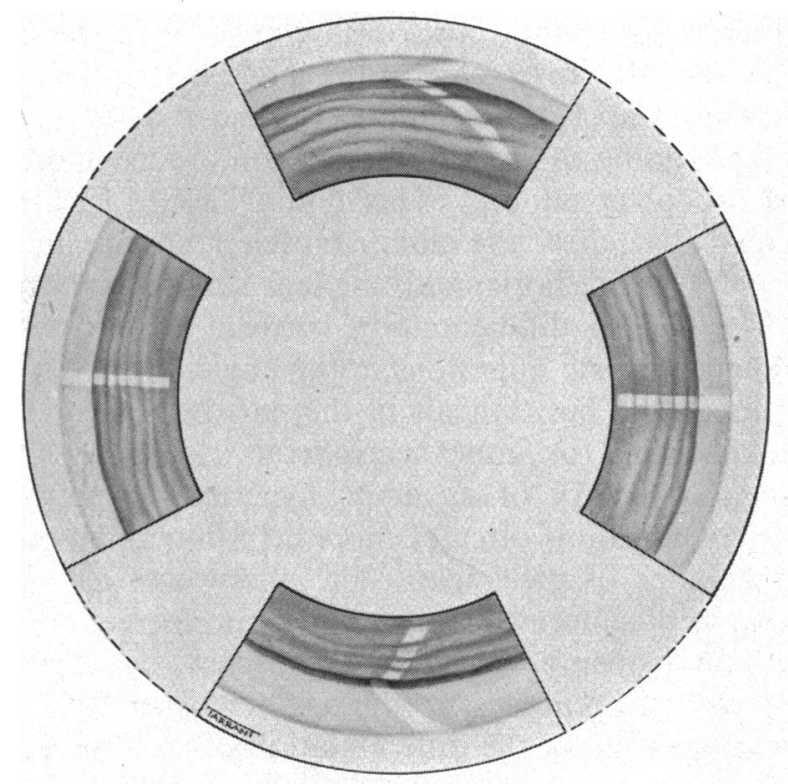

Irido-corneal contact in the upper quadrants is such a very common finding in eyes predisposed to closed-angle glaucoma, even when only the earliest premonitory symptoms are present, that the following conclusions seem justified:

(a) The irido-corneal contact starts, superiorly, long before even the premonitory symptoms appear.

(b) The area of contact spreads slowly downwards, nasally and temporally, as the lens increases in size with increasing age, to cover more and more of the trabeculae. Although the process is one of steady advance, it seems likely that the 
total area occluded varies slightly from hour to hour and minute to minute, depending on the many factors influencing the position of the iris in relation to the trabeculae, especially those affecting the pressure difference on the two sides of the iris diaphragm; physiological or pathological changes in the ciliary body or in the position, size, and shape of the lens may play a part. As the remaining amount of angle allowing drainage of aqueous becomes reduced to a critical level, so these minor (physiological?) variations will, more and more often, cause the irido-corneal contact to extend beyond the limit and the premonitory symptoms of closed-angle glaucoma will appear. If the status quo is soon restored, the subacute "attack" of glaucoma resolves; if not, rising tension and increasing congestion will become established.

Smith (1954) has described the "on-off phenomenon" in closed-angle glaucoma; the angle is seen to open and close as the pupil contracts and dilates on exposure of the eye to a slit-lamp beam which can be quickly switched on and off. This phenomenon would be expected to occur only at the edge of advancing irido-corneal contact.

One of the results reported by Higgitt and Smith (1955) on a reading test, which is sometimes positive in closed-angle glaucoma, lends support to the view that the lower part of the angle is of prime importance. One of their patients (Case1; see their Fig. 1), who showed a rise of intra-ocular pressure when reading in the supine position, added more than $10 \mathrm{~mm}$. $\mathrm{Hg}$ to that rise when he sat up. This finding is not surprising, for Hess (1896) has shown that, after the ciliary muscle contracts in accommodation when the visual axes are horizontal, the lens sinks downwards.

The clinical differentiation between goniosynechiae, in the sense of permanent organic adhesions in the angle, and mere contact of iris and cornea is important but difficult in the narrow angle. On it, mainly, will depend the decision for either peripheral iridectomy or drainage operation. A previous history of an acute hypertensive episode or of many sub-acute rises in tension is often of more help in making the choice than the objective appearance of the angle. The absence of a well-defined edge to a broad expanse of synechiae (the change from mere contact between iris and cornea to definite open-ness of the angle is likewise imperceptible), and the absence of the normal obtuseness of the angle between the plane of the iris and that of the cornea, make the differentiation often impossible.

\section{Effect of Peripheral Iridectomy on Depth of Anterior Chamber}

The difference in the gonioscopic picture before and after peripheral iridectomy is striking, especially when the upper part of the angle has been occluded by contact between the iris and cornea. The opening of the angle which results from the operation is accompanied by an increased depth of the anterior chamber; no objective investigation has been published to show whether this is due to a sinking backwards of the iris alone (the usually accepted explanation) or to a change in position of the lens.

Case 2, a man aged 43.-Measurements of the depth of various parts of the 
anterior chamber were made before and after peripheral iridectomy. The method of Jaeger (1951) was used, although it is less suitable than that of Törnquist (1953). Goniometry (Gradle and Sugar, 1950) would not have yielded the necessary evidence. The central region along the visual axis is susceptible of accurate measurement; to find the depth more peripherally by this technique, points were chosen on the 3,6, and 9 o'clock meridians mid-way between the pupil and the limbus, the pupil being approximately the same size throughout. The mid-points were judged merely by inspection, and the patient's gaze was so directed that the slit-lamp beam fell on them, no adjustment having been made after the setting for the central area.

The results in Case 2 are presented in Fig. 2, where it will be seen that the depth of the anterior chamber in the pupillary area remains almost the same before and after operation, whereas over the iris it increases markedly. In support of the contention that the position of the lens is the same before and after operation may be adduced the fact that the refractive error remained almost identical throughout, there being a slight change in the orientation of $a+0.50$ cylinder.
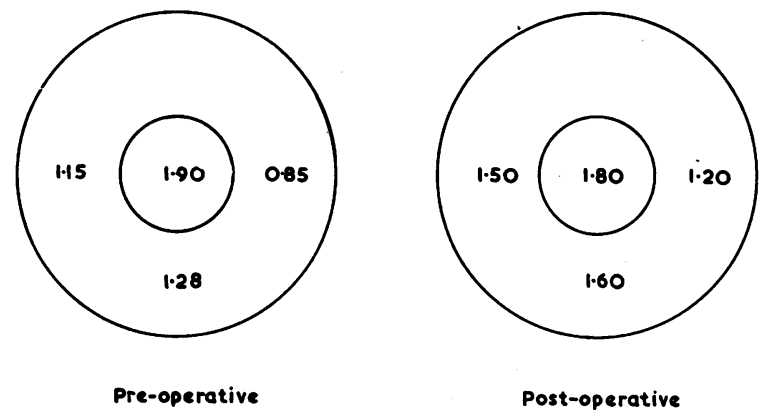

Fig. 2.-Right eye of Case 2, a marn aged 43. The numerals indicate depth, in millimetres, of anterior chamber centrally and half-way between pupil margin and limbus. Inner rings indicate the pupil margin, outer rings the limbus. The operation was peripheral iridectomy.

The calibrations on the instrument allow the depth of the anterior chamber to be expressed in units which are only approximately millimetres; no matter how arbitrary the units of measurements, however, the differences are of undiminished importance. The technique has, of necessity, a small experimental error; each depth recorded was the mean of three separate readings of the instrument, and the greatest of the three pre-operative readings was in no case near the least of the three post-operative ones, so that the differences found are most probably real. On the other hand, the fact that the increase in the depth of the anterior chamber after operation is very slightly less at 6 than at 3 and 9 o'clock could well be attributed to experimental error. Ideally, of course, a series of eyes should be examined and the results statistically analysed.

This patient's left eye had previously had an iridectomy for an acute attack of closed-angle glaucoma, and his symptoms, as well as the results of provocative tests, showed that the right eye had a similar predisposition. 
In order to establish that the iridectomy need not be done only at the 12 o'clock position to produce an increase in the depth of the angle all round, iridectomy was done, in this case, at the 10 o'clock position. The angle widened all round and contact was lost between iris and cornea above as well as elsewhere, with the exception of two extremely small synechiae in the 1 o'clock region. The operation relieved the patient's symptoms.

\section{Causes of Sectoral Variations in Angle Depth}

The factors producing the differences in depth of the various sectors of the angle and the uneven distribution of synechiae must be considered. The possible causes are:

(1) Sectoral differences in the position of origin of the iris from the ciliary body.

(2) Unequal differentiation of sectors of the angle during development.

(3) Orientation of the lens.

(4) Decentration of the pupil.

(5) Uneven peripheral flattening of the cornea.

The first possibility is based on a suggestion by Busacca (1945), but it has not been recorded as accompanying the variations in size known to occur in different segments of the ciliary body (Wolff, 1954).

It seems unlikely that an abnormality in differentiation of the angle during development may account for the variations in its depth, because no evidence for an unusual amount of residual tissue supero-temporally (or a proliferation-Allen, Burian, and Braley, 1955) can be seen gonioscopically when the angle is open. It is interesting to note in this context that the usual eccentricity of the pupil in cases of congenital corectopia is above and lateral to its normal site (Duke-Elder, 1938d).

If the orientation of the lens were the sole explanation, then it must be rotated round an axis running from 8.30 to 2.30 o'clock (in a right eye) on the limbus, the upper part of the equator being the more anterior. In fact, optical experiments have shown that, although a forward tilt of the upper border of the lens does occur, it is slight and inconstant, and that the temporal border always lies behind the nasal border (Duke-Elder, 1938a). Helmholtz (1924a) is in agreement with this observation when he states that the optical axis of the lens meets the cornea at a point that lies outwards and a little downwards from the ophthalmometric axial point, and that the axis of the lens passes to the nasal side of the centre of curvature of the cornea. The axis of the lens, according to Tscherning (1920), lies as much as $0.25 \mathrm{~mm}$. above the centre of curvature of the cornea. The lens is also decentred nasally so that the circumlental space is narrowed in this region (DukeElder, 1938b). The slight tendency for goniosynechiae to occur more on the temporal than the nasal side (Phillips, 1956) is not in accord with the fact that the temporal border of the lens is behind the nasal border; the preponderance of goniosynechiae in the upper part of the angle is more 
marked than would be expected on the basis of a "slight and inconstant" forward tilt of the lens.

The known decentration of the pupil nasally in relation to the optic axis might be expected to result in a greater radial extent of the iris temporally and therefore in greater ballooning.there than elsewhere. The narrowness of the angle above cannot, however, be explained on that basis.

Uneven peripheral flattening of the cornea seems to be the explanation which is in accord with most of the facts. The nearest approach to an axis of symmetry of the cornea " is in a direction about $5^{\circ}$ temporal and slightly below the axis of the eye " (Duke-Elder, 1938c). The distribution of goniosynechiae in closed-angle glaucoma has been found to have a similar axis of symmetry (Phillips, 1956), which runs from 11.30 to 5.30 o'clock on the limbus (in a right eye). And this axis of symmetry, like the corneal astigmatism with the rule in the central area, may in turn be explained by, or associated with, the pressure of the upper lids and especially by the fact that the widest part of the palpebral aperture lies nasal to the centre of the cornea when the eyes are in the primary position; thus the pressure of the upper lid flattens the upper temporal area more than the upper nasal area. Helmholtz (1924b), however, states that the nasal region is affected more than the temporal region by peripheral flattening of the cornea.

\section{Implications of Sectoral Differences in Angle Depth}

It seems likely that the vertical orientation of the oval pupil in an acute attack of closed-angle glaucoma results from the shallowness of the anterior chamber superiorly.

Case 3, a woman aged 53, had previously (in 1947) suffered an acute attack of closed-angle glaucoma in the right eye; the shape of the pupil has not materially altered since the acute attack which responded to intensive miotic therapy. Fig. 3 shows the normal left eye and the affected right eye.
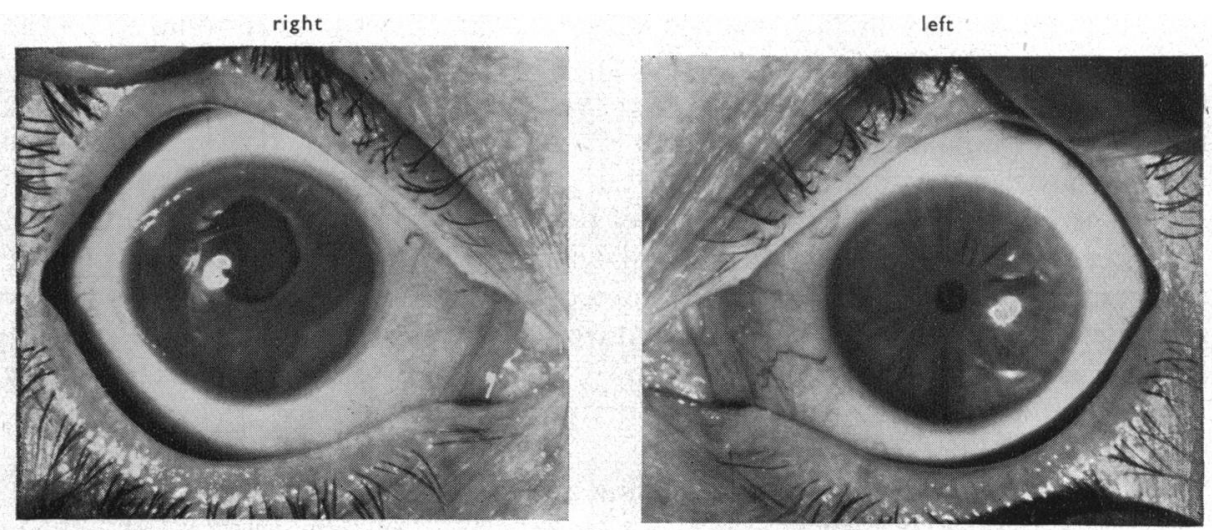

Fig. 3.- Affected right eye and normal left eye of Case 3, a woman aged 53. The great shortening of the iris at 12 o'clock has eliminated the pupillary block and therefore the iris bombé, thus producing resolution of the acute closed-angle glaucoma. 


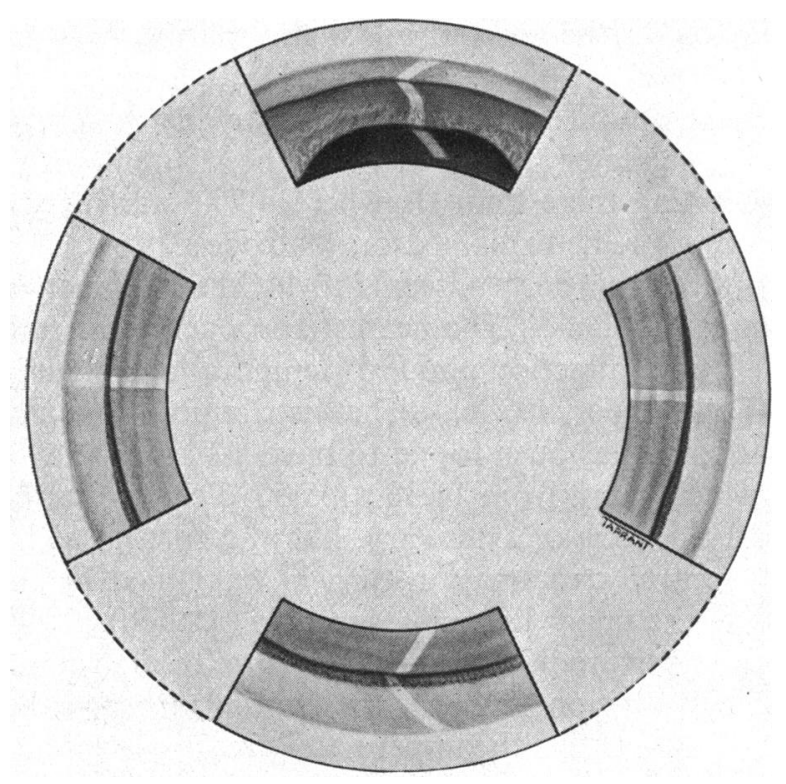

FIG. 4.-Gonioscopic appearances of right eye of Case 3 , which had an acute closedangle glaucoma in 1947 which resolved on intensive eserine. Angle completely closed above but widely open below. Note absence of iris bombé (cf. Fig. 1).

The gonioscopic picture of the right eye, shown in Fig. 4, is very similar to that seen in Fig. 1, with the important exception that there is no actual ballooning of the iris. Measurements show that the pupillary dilation is most marked in the superior region, where the distance between pupil margin and apparent limbus is 42 per cent. of that in the normal left eye, whereas the proportions for the nasal, 6 o'clock, and temporal meridians are 83, 83, and 76 per cent. respectively.

Presumably, at the onset of the acute attack, the contact between iris and cornea had extended so far round the whole circumference of the angle as to start the series of events culminating in an acute episode of glaucoma. Next, the pupil would become vertically oval, as well as semi-dilated, and the sector of the iris at 12 o.clock would become especially shortened. There, evidently (Fig. 4), the irido-corneal contact became quickly converted into a permanent adhesion. The shortening at 12 o'clock must have been great enough to cause the sphincter to ride so far upwards and backwards over the surface of the lens that the resistance to the flow of aqueous through the pupil in that region became negligible, with the result that free communication between posterior and anterior chamber was established there, albeit at the expense of dense synechiae in the 12 o'clock region. This free communication would immediately produce flattening of the iris bombé, thus opening the angle in all areas except superiorly. The eye has therefore succeeded in performing upon itself what would correspond to a central iridectomy. Outflow is now well within normal limits (" $c "=0 \cdot 20$ ). It is surprising that more cases of acute closed-angle glaucoma do not resolve in this way.

\section{Summary}

Attention is drawn to the fact that the angle of the anterior chamber is shallower above than elsewhere. It is suggested that, in eyes which are in danger of ultimately developing closed-angle glaucoma, irido-corneal 
contact starts above long before there are any symptoms, and progresses slowly downwards as the lens increases in size with age. When the free angle becomes reduced nearly to the critical amount for adequate aqueous outflow, the premonitory symptoms are produced by transient increases in the area of trabeculae covered by iris. Provided that there has been no inflammatory reaction in iris or cornea, this contact superiorly may remain for years without being converted into permanent synechiae. These new conceptions make it easier to understand total closure, and hence acute closed-angle glaucoma, resulting from sudden occlusion of the small amount of residual angle left open below.

Measurements of the depth in various parts of the shallow anterior chamber before and after peripheral iridectomy have shown that, while the lens remains stationary, the iris sinks backwards away from the cornea.

It is suggested that the main factor causing the anterior chamber to be shallower above is greater peripheral flattening of the cornea in that region. This, in turn, may be due to pressure of the upper lid.

A case history is described as an example of the occasional resolution of a closed-angle glaucoma by marked shortening of the superior segment of the iris. The aqueous is thus allowed to escape through the pupil at 12 o'clock thereby eliminating the iris bombé. The vertically oval pupil in acute closedangle glaucoma may be an "attempt" by the congested eye to achieve this state.

The clinical details of patients have been made available for publication by the kind permission of Sir Stewart Duke-Elder, Mr. A. J. B. Goldsmith, and Mr. Harold Ridley; their helpful criticism in the preparation of this paper is gratefully acknowledged.

I wish to record my thanks to the staff of the Department of Medical Illustration, Institute of Ophthalmology for producing the drawings (Mr. T. Tarrant) and photographs (Dr. Peter Hansell and Mr. N. Jeffreys).

\section{REFERENCES}

Allen, L., Burian, H. M., and Braley, A. E. (1955). A.M.A. Arch. Ophthal., 53, 783.

BARKAN, O. (1936). Ibid., 15, 101. (1938). Amer. J. Ophthal., 21, 1099.

Busacca, A. (1945). "Elements de gonioscopie". Tipographia Rossolilo, Sao Paulo.

Duke-Elder, S. (1938a). "Text-book of Ophthalmology", vol. 1, p. 118. Kimpton, London.

- (1938b). Ibid., p. 32.

- (1938c). Ibid., p. 742.

- (1938d). Ibid., vol. 2, p. 1316.

- ed. (1955). "Glaucoma. A Symposium ". Blackwell, Oxford.

François, J. (1955). In Duke-Elder (1955), p. 187.

Gradle, H. S., and Sugar, H. S. (1940). Amer. J. Ophthal., 23, 1135.

HelmholtZ, H. L. F. von (1924a). "Treatise on Physiological Optics", vol. 1, p. 356, trans. from 3rd German edition, ed. J. P. C. Southall. Optical Society of America, Wisconsin. (1924b). Ibid., pp. 310, 315.

Hess, C. (1896). Ber. ophthal. Ges. Heidelberg, 25, 41.

Higgitt, A. C., and SMITH, R. (1955). British Journal of Ophthalmology, 39, 103.

JAEGER, W. (1951). Ber. deutsch. Ophthal. Ges., 57, 324.

PHILlIPS, C. I. (1956). British Journal of Ophthalmology, 40, 129.

SMITH, R. (1954). Ibid., 38, 136.

TöRNQUIST, R. (1953). Acta ophthal. (Kbh.), 31, Suppl. 39.

Tscherning: M. (1920). "Physiologic Optics", 3rd ed., p. 45, trans. Carl Weilłand. Keystone, Philadelphia.

WolfF, E. (1954). "The Anatomy of the Eye and Orbit", 4th ed. Lewis, London. 